\title{
Effect of prophylactic TACE on 5-year survival of patients with hepatocellular carcinoma after hepatectomy
}

\author{
SHASHA HE ${ }^{1}$, XIAOTANG FAN ${ }^{2}$, HAILIN MA ${ }^{2}$, HALI XIAERFUHAZI ${ }^{2}$, \\ ALIYA REHATO $^{2}$, JUAN FENG ${ }^{2}$, XIUJIANG SHI ${ }^{2}$ and FANGPING HE ${ }^{1}$ \\ ${ }^{1}$ Department of Infectious Diseases, The Eighth Affiliated Hospital, Sun Yat-Sen University, Shenzhen, Guangdong 518000; \\ ${ }^{2}$ Department of Hepatology, The First Affiliated Hospital of Xinjiang Medical University, Urumqi, Xinjiang 830054, P.R. China
}

Received September 19, 2018; Accepted April 15, 2019

DOI: $10.3892 / \mathrm{ol} .2019 .10517$

\begin{abstract}
The aim of the present study was to establish a prediction index (PI) model for the 5-year survival rate of patients with hepatitis B-related hepatocellular carcinoma (HCC) after radical resection, and to evaluate the effect of prophylactic transcatheter arterial chemoembolization (TACE). A total of 201 patients with hepatitis B-related HCC who had undergone radical hepatic resection at The First Affiliated Hospital of Xinjiang Medical University (Xinjiang, China) were enrolled, and the clinical, pathological and complete follow-up data were collected. Univariate and multivariate Cox regression analyses were performed to identify which clinicopathological factors were considered significant risk factors and the PI model was established based on these factors. The receiver operating characteristic curve was generated, and the area under the curve (0.841) and the cut-off value for PI were calculated. A Kaplan-Meier plot was used for survival analysis and the log-rank test was used to determine differences in survival. Cox regression analysis demonstrated that there were seven independent factors that may have affected the 5-year survival of HCC patients: Neutrophil-to-lymphocyte ratio (NLR), maximum size of tumor (MTS), tumor histological grade (HG), positive resection margin (PRM), microvascular invasion (MVI), the amount of tumor (AT), and antivirus therapy (AVT). A PI model on 5-year survival was established based on these factors, which was PI=0.32 x NLR + 0.39 x HG (high=1, medium $=2$, low $=3)+0.92 \times$ PRM $($ yes $=1$, no $=0)+0.87 \times$ MVI
\end{abstract}

Correspondence to: Dr Fangping He, Department of Infectious Diseases, The Eighth Affiliated Hospital, Sun Yat-Sen University, 3025 Shennan Zhong Road, Shenzhen, Guangdong 518000, P.R. China

E-mail: hefp@sina.com

Abbreviations: PI, prediction index; HCC, hepatocellular carcinoma; ROC, receiver operating characteristic; TACE, transcatheter arterial chemoembolization

Key words: prophylactic intervention, hepatocellular carcinoma, radical resection, survival analysis
$($ yes $=1$, no $=0)+0.73 \times$ AT $($ single $=0$, many $=1)+0.53 \times$ MTS ( $\geq 5 \mathrm{~cm}=1,<5 \mathrm{~cm}=0$ )-0.87 x AVT (yes=1, no=0). PI was an independent predictor for survival, with a cut-off value of 2.75 . For low-risk patients (PI <2.75), there was no significant difference in cumulative survival between TACE and non-TACE. For high-risk patients (PI >2.75), the cumulative survival rates showed significant differences among patients who had received $\geq 3$ TACE procedues, patients who had received $<3$ TACE procedures, and patients who had not undergone TACE. The PI model predicts the 5-year survival rate of patients with hepatitis B-related HCC. For high-risk patients with a PI $>2.75$, if they had received $\geq 3$ prophylactic TACE procedures, they demonstrated a more favorable outcome. For low-risk patients (PI $<2.75$ ) with 1 or 2 risk factors, TACE is recommended 1-2 times after surgery. TACE treatment is not required for low-risk patients without any risk fctors. These results may contribute to the decision-making process for whether prophylactic intervention is recommended after radical resection of HCC.

\section{Introduction}

Hepatocellular carcinoma (HCC) is one of the most common malignancies, with $\sim 750,000$ people diagnosed with HCC per year (1), of which nearly $55 \%$ occur in China (2). There are between 600,000 and 700,000 HCC-associated deaths each year worldwide (3), with 360,000 deaths in China alone (4). China has a high incidence of hepatitis B virus (HBV) infections and chronic $\mathrm{HBV}$ infection is one of the principal causes of HCC.

Surgical resection is the most effective method for treating patients with HCC at present. For resectable HCC, surgery is the first consideration. However, a high postoperative recurrence rate and metastasis rate limit the long-term survival of patients, even after radical surgery $(5,6)$. In addition, HCC patients with HBV infection often exhibit cirrhosis, and $\mathrm{HCC}$ resection is limited by the severity of cirrhosis (7). In patients with persistent HBV infection, there are certain pathological factors that influence postoperative recurrence (including tumor size, microvascular invasion (MVI), surgical margin, foci, biological characteristics and the number of tumors); therefore, in theory, almost all HCC cases are likely to recur after surgery, and intrahepatic and extrahepatic metastases 
can be difficult to treat via surgery (8). Even if the tumor is completely resected, small cancerous lesions may exsit in the liver that cannot be detected by preoperative or intraoperative radiology. Postoperative recurrence and metastasis of HCC may seriously decrease a patient's long-term survival $(5,6)$.

Local treatment of the liver may be more effective than systemic therapies (5). The prophylactic transcatheter arterial chemoembolization (TACE) method is the most commonly used local treatment, and is widely used clinically to improve the efficacy of HCC surgery. Hepatic artery angiography and subsequent lipiodol computed-tomography (CT) examination during prophylactic TACE treatment are the most sensitive methods to detect residual cancer and early recurrence at an early stage (9). These methods allow for timely diagnosis and treatment of postoperative residual cancer or early recurrence (10) and therefore, adoption of TACE has become more widespread. It is strongly recommended that patients undergoing radical resection of HCC are treated with TACE in a timely manner after surgical resection $(11,12)$. However, to the best of our knowledge, there are currently no studies evaluating the effectiveness of postoperative prophylactic TACE treatment for which a large-scale randomized controlled trial is preferable.

In the present study, a prediction index (PI) model of the 5-year survival rate of patients with hepatitis B-related HCC after radical resection was established and the effect of prophylactic TACE on patient survival was determined. The model may improve prognosis through individualized prophylactic TACE intervention after radical resection of HCC.

\section{Materials and methods}

Patients. The medical records of patients with hepatitis B-related HCC, that had received radical resection in The First Affiliated Hospital of Xinjiang Medical University (Urumqi, China) from October 2007 to December 2010 were retrospectively reviewed. There were 201 patients in the final cohort, of which 130 were male and 71 were female. The age range of the cohort was 38-69 years with a mean age of $52 \pm 8$ years. Informed consent was obtained from each patient and the study was approved by The Ethics Review Board of The First Affiliated Hospital of Xinjiang Medical University.

Inclusion and exclusion criteria. The inclusion criteria for patients were: i) Patients were in good general health and had no obvious heart, lung, kidney or other vital organ diseases; ii) had a liver function grade A or B; iii) had chronic hepatitis B infection based on the Guidelines for Prevention and Treatment of Chronic Hepatitis B (2005 Edition) (13); and iv) had surgical radical resection. Radical resection was considered if the patients had: i) Complete resection of the tumor; ii) no residual cancer; iii) had $\leq 3$ distinct tumours; iv) no tumor embolus in the portal vein or branches, primary hepatic duct or branches or in the hepatic vein or the inferior vena cava; and v) no lymph node or distant metastases (14).

The exclusion criteria for patients were: i) Presence of other malignant tumors; ii) other types of viral hepatitis, drug-induced hepatitis, alcoholic liver diseases, autoimmune liver diseases or hereditary metabolic liver diseases; iii) decompensated cirrhosis; iv) a liver function grade of C; v) major underlying diseases (cardiovascular diseases and respiratory diseases); or vi) incomplete information.

Follow-ups. Patients were followed up by telephone, clinical visits and letters. The time of surgery was considered as the starting point and 5 years after the operation was considered as end point. Outcome measurement included survival or mortality. At baseline, patient's age, sex, preoperative examinations (immunity markers, tumor markers, HBV DNA load, abdominal CT angiography), surgical procedures, postoperative examinations (liver function, renal function, rountine blood test results), pathological biopsy report, anti-virus therapy (AVT), and prophylactic TACE treatment were recorded. The lower value was extracted if there were two inconsistent postoperative biochemical blood analyses. The maximum diameter of a single tumor was defiened as the largest diameter on the largest cross-sectional area. The sum of the maximum diameters of all the tumors was termed the 'diameter of multiple tumors'. The amount of tumor (AT) was determined based on preoperative imaging, including $\mathrm{C} \backslash \backslash$ magnetic resonance imaging and contrast-enhanced ultrasound. Detailed variables are listed in Table I.

The surgical margin was considered positive if there were: i) Slightly infiltrating lesions in the surgical margins; ii) abnormal margins or precancerous lesions; or iii) the margins of the resected lesions were $<0.5 \mathrm{~mm}$.

TACE. TACE was performed 2-12 weeks after surgery and the dosage regimen was determined based on the angiographic results. If tumor blood vessel staining was not observed during surgery, the proper hepatic artery would be infused with $750 \mathrm{mg}$ of 5-fluorouracil and $60 \mathrm{mg}$ of cisplatin, and subsequently embolized with a suspension of $3 \mathrm{ml}$ of super-liquefied lipiodol and $6 \mathrm{mg}$ of mitomycin. The procedure was repeated once after 6-8 weeks in certain cases with risk factors, such as MVI. If tumor blood vessel staining was observed during surgery, the dose was determined according to the size and number of lesions and the patient's condition. CT examination was performed 6 weeks after TACE treatment to evaluate the intrahepatic condition.

Statistical analysis. SPSS 19.0 software (IBM Corp., Armonk, NY, USA) was used for statistical analysis. The univariate and multivariate Cox regression analyses were performed to identify the significant risk factors and the PI model was established based on the signficant factors. A receiver operating characteristic (ROC) curve was drawn and the area under the receiver operating characteristic curve (AUROC) was determined. The Youden index at the maximum corresponding values was used to calculate the PI threshold. Kaplan-Meier survival analysis was used to analyze the cumulative survival and a Log-Rank test was used to determine differences in survival. $\mathrm{P}<0.05$ was considered to indicate a statistically significant difference.

\section{Results}

Patient characteristics. A total of 201 HCC cases were included in the present study. Among the 201 patients, there 
Table I. Univariate Cox regression analysis of the 5-year survival rate of patients with hepatitis B-related hepatocellular carcinoma.

\begin{tabular}{lccccccc}
\hline Variables & Regression $\beta$ & Standard error & Wald & Levels of freedom & P-value & Odds ratio & $95 \%$ CI \\
\hline Hepatitis B DNA & 0.44 & 0.23 & 3.85 & 1 & 0.050 & 1.56 & $1.00-2.43$ \\
Prealbumin & -0.01 & 0.00 & 4.76 & 1 & 0.029 & 0.99 & $0.98-1.00$ \\
PTA & -0.02 & 0.01 & 6.54 & 1 & 0.011 & 0.98 & $0.96-1.00$ \\
Tumor envelope & 0.45 & 0.22 & 4.08 & 1 & 0.043 & 1.57 & $1.01-2.43$ \\
AVT & -0.56 & 0.23 & 6.00 & 1 & 0.014 & 0.57 & $0.36-0.89$ \\
NLR & 0.44 & 0.10 & 18.27 & 1 & $<0.001$ & 1.55 & $1.27-1.89$ \\
MVI & 0.87 & 0.23 & 14.57 & 1 & $<0.001$ & 2.38 & $1.52-3.71$ \\
AT & 0.73 & 0.221 & 10.75 & 1 & 0.001 & 2.07 & $1.34-3.21$ \\
PRM & 0.89 & 0.21 & 17.26 & 1 & $<0.001$ & 2.43 & $1.60-3.68$ \\
MTS & 0.68 & 0.22 & 9.24 & 1 & 0.002 & 1.97 & $1.27-3.05$ \\
HG & 0.38 & 0.14 & 7.02 & 1 & 0.008 & 1.46 & $1.10-1.92$
\end{tabular}

CI, confidence interval; PTA, prothrombin activity; AVT, antivirus therapy; NLR, neutrophil-to-lymphocyte ratio; MVI, microvascular invasion; AT, the amount of tumor; PRM, positive resection margin; MTS, the maximum size of tumor; HG, histological grade.

Table II. Multivariate Cox regression analysis of 5-year survival in patients with hepatitis B-related hepatocellular carcinoma.

\begin{tabular}{lccrrrrr}
\hline Variables & Regression $\beta$ & Standard error & Wald & Levels of freedom & P-value & Odds ratio & 95\% CI \\
\hline NLR & 0.32 & 0.11 & 8.80 & 1 & 0.003 & 1.38 & $1.11-1.70$ \\
AVT & -0.87 & 0.24 & 13.04 & 1 & $<0.001$ & 0.42 & $0.26-0.67$ \\
MVI & 0.73 & 0.24 & 9.09 & 1 & 0.003 & 2.08 & $1.29-3.35$ \\
AT & 0.46 & 0.23 & 4.03 & 1 & 0.045 & 1.58 & $1.01-2.46$ \\
PRM & 0.92 & 0.22 & 16.80 & 1 & $<0.001$ & 2.50 & $1.61-3.88$ \\
MTS & 0.53 & 0.23 & 5.42 & 1 & 0.020 & 1.69 & $1.09-2.63$ \\
HG & 0.39 & 0.15 & 6.91 & 1 & 0.009 & 1.47 & $1.10-1.97$ \\
\hline
\end{tabular}

The corrected factors included smoking, sex, prealbumin, alanine aminotransferase, albumin, triglycerides, hepatitis B DNA, liver function grades, cirrhosis, total bilirubin, prothrombin activity, $\gamma$ glutamyl transpeptidase and tumor envelope. CI, confidence interval; AVT, antivirus therapy; NLR, neutrophil-to-lymphocyte ratio; MVI, microvascular invasion; AT, the amount of tumor; PRM, positive resection margin; MTS, the maximum size of tumor; HG, histological grade.

were 130 males $(64.7 \%)$ and 71 females $(35.3 \%)$, with an age range of 38-69 years (mean age, $52 \pm 8$ years). The survival time range was 7-60 months. Of the 201 patients, 81 individuals succumbed to the cancer $(40.3 \%)$ and 120 individuals survived for a minimum of 5 years $(59.7 \%)$. A total of 95 patients were treated with TACE.

Univariate Cox regression analysis. Univariate Cox regression analysis showed that among the 34 related indicators, 11 showed significant differences, including Hepatitis B DNA, pre-albumin, prothrombin activity, AVT, neutrophil-to-lymphocyte ratio (NLR), MVI, the maximum size of tumor (MTS), AT, tumor histological grade (HG), tumor envelope and positive resection margin (PRM). The above factors may have affected the patients' survival and prognosis $(\mathrm{P}<0.05$; Table I).

Multivariate Cox regression analysis. All indicators in univariate analysis were ranked based on the P-values from the smallest to the largest. The first 20 indexes were further analyzed with the multivariate Cox risk regression model. After adjusting the confounding variables, seven independent factors were identified. As shown in Table II, there were six independent risk factors (NLR, MTS, HG, PRM, MVI, and AT) that may have increased the 5-year mortality risk of patients and 1 independent protective factor (AVT). The relative odd ratios (ORs) of NLR, MTS, HG, PRM, MVI, and AT were 1.38, $1.69,1.47,2.50,2.08$ and 1.58 . With a 1-unit increase in NLR, the risk of succumbing to the cancer within 5 years was be increased by $38 \%$. Each 1-unit reduction in HG increased the probability of mortality by $47 \%$ within 5 years. AVT was the independent factor for 5-year mortality reduction (OR, 0.42).

Cox risk regression analysis of postoperative TACE treatment. Cox regression univariate and multivariate analyses of postoperative TACE treatment were performed. In the adjusted Cox regression model, postoperative TACE treatment demonstrated a significant difference, suggesting that TACE may have a significant effect on the 5-year survival of these patients (Table III). The corrected OR value was 0.51 , 
Table III. Cox risk proportional regression analysis of postoperative TACE treatment.

\begin{tabular}{|c|c|c|c|c|c|c|c|}
\hline Variables & Regression $\beta$ & Standard error & Wald & Levels of freedom & P-value & Odds ratio & $95 \% \mathrm{CI}$ \\
\hline Before adjustment & -0.46 & 0.23 & 4.16 & 1 & 0.041 & 0.63 & $0.40-0.98$ \\
\hline After adjustment & -0.67 & 0.24 & 8.14 & 1 & 0.004 & 0.51 & $0.32-0.81$ \\
\hline
\end{tabular}

The corrected factors included age, sex, smoking history, prealbumin, albumin, total bilirubin, serum creatinine, prothrombin activity, $\gamma$ glutamyl transpeptidase, intraoperative blood loss, low density lipoprotein, alanine aminotransferase, aspartate aminotransferase, triglycerides, Hepatitis B DNA, liver function grades, cirrhosis, tumor envelope, and TACE treatment. CI, confidence interval; TACE, transcatheter arterial chemoembolization.

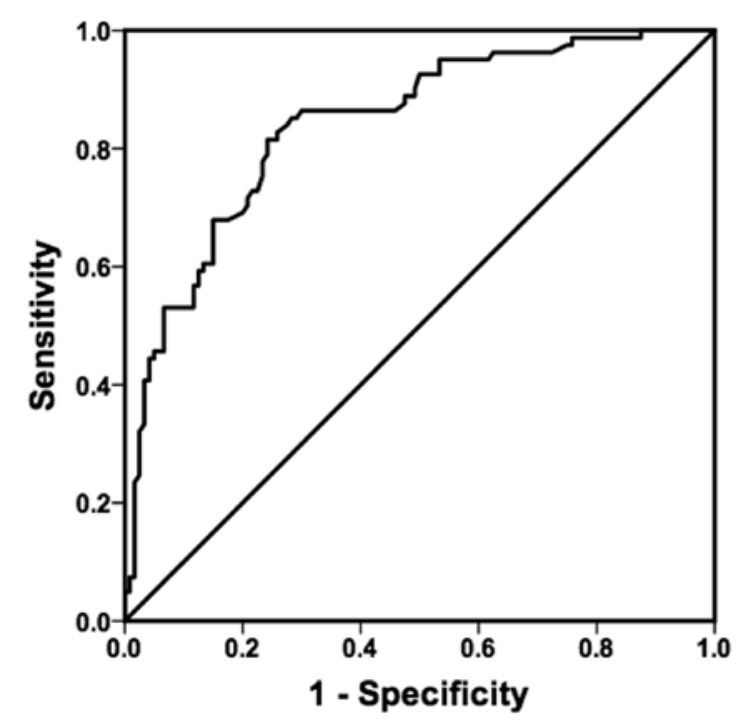

Figure 1. Receiver operating characteristic curve of the prediction index value in predicting 5-year survival of patients with hepatitis B-related hepatocellular carcinoma. The area under the curve was 0.841 (95\% confidence interval, 0.786-0.897). The cut-off value was 2.75 .

thus, the postoperative risk of mortality within 5 years was 1.96 times greater for patients who did not receive TACE treatment compared with patients that did receive it $(\mathrm{P}<0.05$; Table III).

Establishment of the 5-year survival prediction model. The PI model developed, based on the seven significant factors identified by the multivariate analysis, was: $\mathrm{PI}=0.32 \times \mathrm{NLR}+0.39$ x HG (high=1, medium=2, low=3) + 0.92 x PRM (yes=1, no=0) $+0.87 \times$ MVI $($ yes $=1$, no $=0)+0.73 \times$ AT $($ single $=0$, many $=1)+0.53 \times$ MTS $(\geq 5 \mathrm{~cm}=1,<5 \mathrm{~cm}=0)-0.87 \times$ AVT (yes $=1$, no=0). The PI values of the patients in the present study ranged between 0.72 and 4.78 . The larger the PI value, the higher the mortality rate within 5 years; the smaller the PI value, the lower the mortality rate within 5 years. Fig. 1 shows that the AUROC of PI was 0.841, suggesting that the predictive value of PI was moderate. The PI cut-off value, which corresponded to the maximum Youden index, was 2.75. The PI value was analyzed by the Cox risk regression model. After adjusting the factors, the PI values became independent predictors, and for each additional PI unit, the risk of death within 5 years was increased by 1.72 times $(\mathrm{P}<0.05$; Table IV).
Survival analysis. The patients with a PI value $>2.75$ were defined as the high-risk group and those with a PI value $<2.75$ as the low-risk group. Kaplan-Meier survival analysis showed that the 1, 3 and 5-year cumulative survival rate of the group treated with TACE was 97.6, 95.2 and $90.5 \%$ respectively. In the low-risk group (PI <2.75), the 1, 3 and 5-year cumulative survival rate was $95.2,85.5$ and $83.9 \%$, respectively, for patients who did not receive TACE treatment (Fig. 2). However, there was no significant difference between the two groups in the cumulative survival rates at all stages $(\mathrm{P}>0.05)$. For patients in the high risk group, the cumulative survival rate at all stages showed significant differences between patients who received $>3$ TACE treatments compared with that received $<3$ TACE treatments $(\mathrm{P}<0.05$; Fig. 3$)$. The cumulative survival rates at 1, 3 and 5-years were as follows: $\geq 3$ TACE treatments were: $100 ; 80.0$; and $53.3 \%$ respectively, for patients who received $<3$ TACE treatments: 81.8 ; 40.9; and $36.4 \%$ repectively, and for patients who did not receive TACE treatment: 73.3; 13.3; and $13.3 \%$ respectively $(\mathrm{P}<0.05$; Fig. 3$)$.

\section{Discussion}

HCC is a blood vessel-rich tumor enabling easier extravasation into blood vessels, and metastasis to the hepatic sinusoids, and portal vein through the formation of micro-venous intrahepatic metastases (15). Therefore, MVI is often considered a major risk factor for postoperative recurrence and intrahepatic metastasis (15-18). MVI is additionally a risk factor for early recurrence (19). Postoperative TACE therapy in MVI-positive patients can prevent the establishment of a blood supply, preventing or at least limiting the proliferation of residual tumor cells, reducing the risk of early recurrence and metastasis, and thus, increasing disease-free and overall survival.

The size and number of tumors are important factors affecting postoperative recurrence (20-24) as they can reflect the aggressiveness of the tumor. Large tumors, especially tumors larger than $5 \mathrm{~cm}$, significantly increases the risk of postoperative recurrence. Previous studies have demonstrated that the incidence of intrahepatic metastases and portal vein invasion in tumors larger than $5 \mathrm{~cm}$ was significantly higher compared with those smaller than $5 \mathrm{~cm}$ (25), and the larger the tumor, the earlier postoperative recurrence occurred (26).

Tumor diameter, number, positive surgical margins, and MVI were the risk factors that significantly influenced postoperative recurrence and metastasis. For patients with these 
Table IV. Cox risk proportional regression analysis of PI model predicting 5-year survival in patients with hepatitis B-related hepatocellular carcinoma.

\begin{tabular}{lccccccr}
\hline Variables & Regression $\beta$ & Standard error & Wald & Levels of freedom & P-value & Odds ratio & 95\% CI \\
\hline Before adjustment & 1.00 & 0.12 & 66.78 & 1 & 0.000 & 2.72 & $2.14-3.46$ \\
After adjustment & 1.00 & 0.12 & 66.78 & 1 & 0.000 & 2.72 & $2.14-3.46$ \\
\hline
\end{tabular}

The corrected factors included age, sex, smoking history, prealbumin, albumin, total bilirubin, serum creatinine, prothrombin activity, $\gamma$ glutamyl transpeptidase, intraoperative blood loss, low density lipoprotein, alanine aminotransferase, aspartate aminotransferase, triglycerides, Hepatitis B DNA, liver function grades, cirrhosis, tumor envelope, and TACE treatment. CI, confidence interval; TACE, transcatheter arterial chemoembolization.

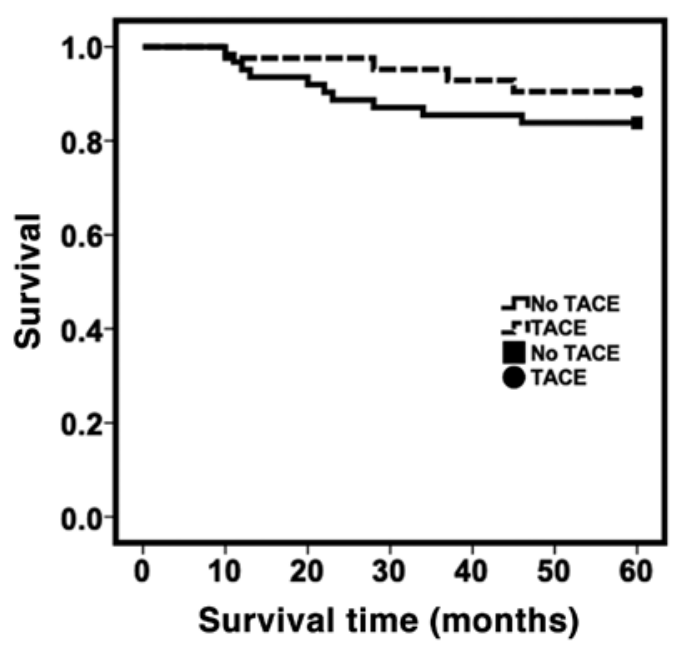

Figure 2. Survival curve of postoperative TACE and non-TACE treatment in the low risk group. Kaplan-Meier survival analysis was used to analyze the cumulative survival and the Log-Rank test was used to determine differences in survival. The 1-year, 3-year and 5-year cumulative survival rates of the TACE group were 97.6, 95.2 and $90.5 \%$, respectively. The 1-year, 3-year and 5-year cumulative survival rates of non-TACE group were 95.2, 85.5 and $83.9 \%$. However, there was no significant difference in survival curve distribution and cumulative survival rate between the two groups $(\mathrm{P}>0.05)$. TACE, transcatheter arterial chemoembolization.

risk factors, TACE should be performed at 2-12 weeks after surgery based on the results of the multivariate Cox regression analysis. To determine the individualized TACE treatment regimen for patients, a PI model was established. The Cox regression analysis showed that a one unit increase in PI value increased the risk of mortality withing 5 years by 1.72 times. The final Cox multivariate regression model was refined by accounting for the number of times TACE was performed. For high-risk patients with a PI $>2.75$, the survival curves showed that $\geq 3$ TACE treatments significantly improved overall survival. Based on the PI model, the early use of prophylactic interventional therapy for high-risk HCC patients after radical surgery may improve the 5-year survival rate. The PI model may provide evidence for the early intervention treatment after radical resection of liver cancer.

For the low-risk group, although the cumulative survival rates in each phase of the TACE group were higher than those in the non-TACE group, no statistical significance was found. Based on the data, it may be hypothesized that there are certain risk factors (such as positive surgical margins,

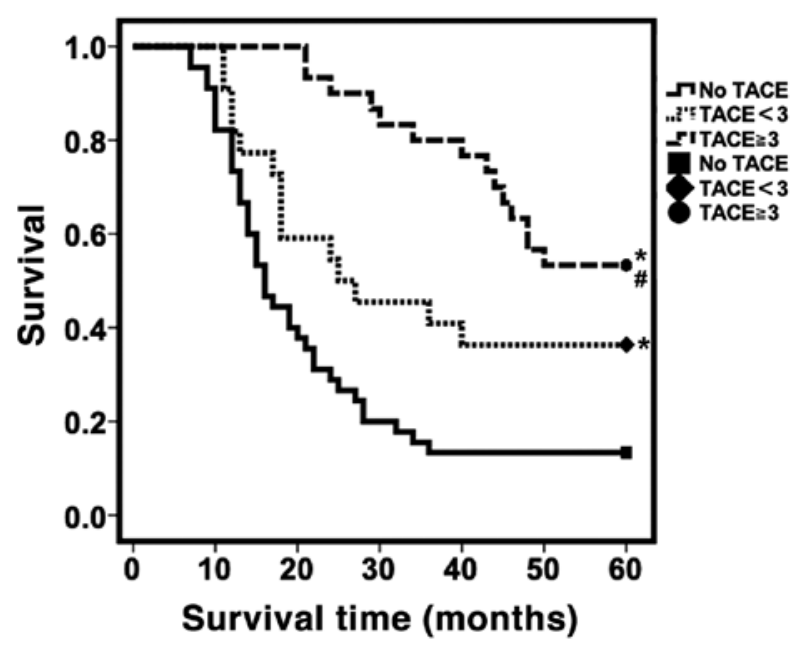

Figure 3. Distribution of survival curves for patients treated with TACE $\geq 3$ times, $<3$ times or no TACE treatment for the high-risk group. Kaplan-Meier survival analysis was used to analyze the cumulative survival and the log-rank test was used to determine differences in survival. In the high-risk group, patients who received $\geq 3$ TACE treatments, TACE $<3$ times or no TACE treatment had significant differences in survival curve distribution and the cumulative survival rates at each stage. The 1 year, 3 years and 5 years cumulative survival rates were: TACE $\geq 3$ : 100, 80.0, 53.3\%; TACE <3: 81.8, 40.9, 36.4\%; no TACE treatment: $73.3,13.3$, 13.3\%. ${ }^{*} \mathrm{P}<0.05$ compared with no TACE treatment at all stages. ${ }^{*} \mathrm{P}<0.05$ compared with TACE $<3$ times at all stages. TACE, transcatheter arterial chemoembolization.

larger tumor volume, multiple lesions and microvascular thrombosis) which contribute to metastasis (15-26). The postoperative TACE treatment may help eliminate these risk factors and improve survival. For those with limited tumor or no tumor thrombus, radical resection is usually achieved and TACE treatment in not needed (13). Regular follow-ups should be performed. Therefore, based on the results of the present study, it is recommended that low-risk patients receive 1-2 TACE treatments after surgery. Multiple TACE treatments may increase postoperative liver load, prolong the postoperative immunosuppressive period, and thus, reduce the quality of life $(10,11)$.

The risk factors that affected the 5-year survival in hepatitis B-related HCC were investigated and a predictive model to identify the high-risk groups with low 5-year survival rates after HCC radical resection was developed in the present study. The prediction model demonstrated that 1-3 TACE treatments with TACE for high-risk patients significantly improved the 
5-year survival rate. For the low-risk group, there was no significant difference in the 5-year survival rate between the TACE group and non-TACE group. These results suggest that individualized early TACE treatment after radical HCC surgery is recommended.

The present study has certain limitations. The sample size was relatively small and recurrence-free survival was not analyzed. In future studies, larger sample sizes will be required to optimize the PI model and to evaluate the recurrence-free survival.

In conclusion, postoperative prophylactic TACE should be considered after radical resection of HCC. Based on the PI model developed in the present study, $\geq 3$ prophylactic TACE treatments will have the most significant improvements in patient outcome for high-risk patients (PI >2.75), and for low-risk patients or patients with $\leq 2$ risk factors ( $\mathrm{PI}<2.75$ ), $<3$ prophylactic TACE treatments is recommended after surgery. TACE treatment is not recommended for low-risk patients without any risk factors. The PI model developed in the present study may be used as a guide to determine whether treatment with TACE is recommended or a per-patient basis following radical resection of $\mathrm{HCC}$.

\section{Acknowledgements}

Not applicable.

\section{Funding}

The present study was supported by The National Natural Science Foundation of China (grant no. 81360138).

\section{Availability of data and materials}

The data used and analyzed during the present study are available from the corresponding author on reasonable request.

\section{Authors' contributions}

FH designed the study and reviewed the manuscript. $\mathrm{SH}$, XF, HM, HX and AR performed the experiments. JF and XS provided help with data analysis and critically revised the manuscript. SH analyzed data and wrote the manuscript. All authors have read and approved the final manuscript.

\section{Ethical approval}

All procedures performed in the present study involving human participants were approved by The Ethics Review Board of The First Affiliated Hospital of Xinjiang Medical University and conducted in accordance with the ethical standards of the Ethics Review Board of The First Affiliated Hospital of Xinjiang Medical University and with the 1964 Helsinki declaration and its later amendments or comparable ethical standards. Informed consent was obtained from each patient.

\section{Patient consent for publication}

Not applicable.

\section{Competing interests}

The authors declare that they have no competing interests.

\section{References}

1. Jemal A, Bray F, Center MM, Ferlay J, Ward E and Forman D: Global cancer statistics. CA Cancer J Clin 61: 69-90, 2011.

2. Zhang XF, Qi X, Meng B, Liu C, Yu L, Wang B and Lv Y: Prognosis evaluation in alpha-fetoprotein negative hepatocellular carcinoma after hepatectomy: Comparison of five staging systems. Eur J Surg Oncol 36: 718-724, 2010.

3. Amarapurkar D, Han KH, Chan HL and Ueno Y; Asia-Pacific Working Party on Prevention of Hepatocellular C: Application of surveillance programs for hepatocellular carcinoma in the Asia-Pacific Region. J Gastroenterol Hepatol 24: 955-961, 2009.

4. El-Serag HB and Rudolph KL: Hepatocellular carcinoma: Epidemiology and molecular carcinogenesis.Gastroenterology 132: 2557-2576, 2007.

5. Lai EC and Lau WY: The continuing challenge of hepatic cancer in Asia. Surgeon 3: 210-215, 2005.

6. Lau WY, Lai EC, Leung TW and Yu SC: Adjuvant intra-arterial iodine-131-labeled lipiodol for resectable hepatocellular carcinoma: A prospective randomized trial-update on 5-year and 10-year survival. Ann Surg 247: 43-48, 2008.

7. Cucchetti A, Zanello M, Cescon M, Ercolani G, Del Gaudio M, Ravaioli M, Grazi GL and Pinna AD: Improved diagnostic imaging and interventional therapies prolong survival after resection for hepatocellular carcinoma in cirrhosis: The university of bologna experience over 10 years. Ann Surg Oncol 18: 1630-1637, 2011.

8. Xie H, Wang H, An W, Ma W, Qi R, Yang B, Liu C, Gao Y, Xu B and Wang W: The efficacy of radiofrequency ablation combined with transcatheter arterial chemoembolization for primary hepatocellular carcinoma in a cohort of 487 patients. PLoS One 9: e89081, 2014.

9. Lin Z, Ren Z and Xia J: Appraisal of postoperative transcatheter arterial chemoembolization (TACE) for prevention and treatment of hepatocellular carcinoma recurrence. Zhonghua Zhong Liu Za Zhi 22: 315-317, 2000 (In Chinese).

10. Lee JK, Chung YH, Song BC, Shin JW, Choi WB, Yang SH, Yoon HK, Sung KB, Lee YS and Suh DJ: Recurrences of hepatocellular carcinoma following initial remission by transcatheter arterial chemoembolization. J Gastroenterol Hepatol 17: 52-58, 2002.

11. Portolani N, Coniglio A, Ghidoni S, Giovanelli M, Benetti A, Tiberio GA and Giulini SM: Early and late recurrence after liver resection for hepatocellular carcinoma: Prognostic and therapeutic implications. Ann Surg 243: 229-235, 2006.

12. Samuel M, Chow PK, Chan Shih-Yen E, Machin D and Soo KC: Neoadjuvant and adjuvant therapy for surgical resection of hepatocellular carcinoma. Cochrane Database Syst Rev 21: CD001199, 2009

13. Association HBaIDBoCM: Guidelines for prevention and treatment of chronic hepatitis B (2010 Edition). Chin J Hepatology Electronic Edition 3: 40-56, 2011.

14. Sun HC, Zhang W, Qin LX, Zhang BH, Ye QH, Wang L, Ren N, Zhuang PY, Zhu XD, Fan J and Tang ZY: Positive serum hepatitis $\mathrm{B}$ e antigen is associated with higher risk of early recurrence and poorer survival in patients after curative resection of hepatitis B-related hepatocellular carcinoma. J Hepatol 12: 684-690, 2007.

15. Roayaie S, Blume IN, Thung SN, Guido M, Fiel MI, Hiotis S, Labow DM, Llovet JM and Schwartz ME: A system of classifying microvascular invasion to predict outcome after resection in patients with hepatocellular carcinoma. Gastroenterology 137: 850-855, 2009.

16. Ardiles V, Sánchez Clariá R, Mazza OM, Ciardullo MA, Pekolj J and De Santibañes E: Prognostic factors after resection of hepatocellular carcinoma in the non-cirrhotic liver: Presentation of 51 cases. Cir Esp 87: 148-154, 2010 (In Spanish).

17. Sumie S, Kuromatsu R, Okuda K, Ando E, Takata A, Fukushima N, Watanabe Y, Kojiro M and Sata M: Microvascular invasion in patients with hepatocellular carcinoma and its predictable clinicopathological factors. Ann Surg Oncol 15: 1375-1382, 2008.

18. Tian YH, Li JD, Peng Y, Li QL, Li DX and Li Q: Postoperative survival analysis of patients without liver cirrhosis. Chin J Digestive Surg 10: 305-306, 2011. 
19. Yamamoto J, Kosuge T, Takayama T, Shimada K, Yamasaki S, Ozaki H, Yamaguchi N and Makuuchi M: Recurrence of hepatocellular carcinoma after surgery. Br J Surg 83: 1219-1222, 1996.

20. Geschwind JF, Kudo M, Marrero JA, Venook AP, Chen XP, Bronowicki JP, Dagher L, Furuse J, Ladrón de Guevara L, Papandreou C, et al: TACE treatment in patients with sorafenib-treated unresectable hepatocellular carcinoma in clinical practice: Final analysis of GIDEON. Radiology 279: 630-640, 2016

21. Hua YF, Li CD, Qiu F, Yu WM, Wu SD, Zhang GJ, Peng T and Yang HT: Transcatheter arterial chemoembolization after liver resection for hepatocellular carcinoma with portal vein tumor thrombus. Chin J Hepatobiliary Surg 18: 357-360, 2012 (In Chinese)

22. Shah SA, Cleary SP, Wei AC, Yang I, Taylor BR, Hemming AW, Langer B, Grant DR, Greig PD and Gallinger S: Recurrence after liver resection for hepatocellular carcinoma: Risk factors, treatment and outcomes. Surgery 141: 330-339, 2007.

23. Tsurusaki M and Murakami T: Surgical and locoregional therapy of HCC: TACE. Liver Cancer 4: 165-175, 2015.
24. Zhu Q, Qiao GL, Yan JJ, Wu MC and Yan YQ: Prognosis after resection of early hepatocellular carcinoma in HBV-related cirrhotic patients. Chin J Hepatobiliary Surg 20: 258-264, 2014.

25. Otto G, Heuschen U, Hofmann WJ, Krumm G, Hinz U and Herfarth C: Survival and recurrence after liver transplantation versus liver resection for hepatocellular carcinoma: A retrospective analysis. Ann Surg 227: 424-432, 1998

26. Pawlik TM, Delman KA, Vauthey JN, Nagorney DM, Ng IO, Ikai I, Yamaoka Y, Belghiti J, Lauwers GY, Poon RT and Abdalla EK: Tumor size predicts vascular invasion and histologic grade: Implications for selection of surgical treatment for hepatocellular carcinoma. Liver Transpl 11: 1086-1092, 2005.

(i) $($ ) This work is licensed under a Creative Common Attribution-NonCommercial-NoDerivatives 4.0 International (CC BY-NC-ND 4.0) License. 